Article

\title{
Reusable Polymer-Supported Terpyridine Palladium Complex for Suzuki-Miyaura, Mizoroki-Heck, Sonogashira, and Tsuji-Trost Reaction in Water
}

\section{Toshimasa Suzuka *, Kosuke Kimura and Takuya Nagamine}

Department of Chemistry, Biology \& Marine Science, University of the Ryukyus, Okinawa 903-0213, Japan; E-Mails: e073309@eve.u-ryukyu.ac.jp (K.K.); e073332@eve.u-ryukyu.ac.jp (T.N.)

* Author to whom correspondence should be addressed; E-Mail: suzuka@ @ci.u-ryukyu.ac.jp; Tel.: +81-98-895-8531; Fax: +81-98-895-8531.

Received: 10 January 2011; in revised form: 8 February 2011 / Accepted: 9 March 2011 / Published: 14 March 2011

\begin{abstract}
A novel heterogeneous transition-metal catalyst comprising a polymer-supported terpyridine palladium(II) complex was prepared and found to promote the Suzuki-Miyaura, Mizoroki-Heck, Sonogashira, and Tsuji-Trost, reactions in water under aerobic conditions with a high to excellent yield. The catalyst was recovered by simple filtration and directly reused several times without loss of catalytic activity.
\end{abstract}

Keywords: palladium; terpyridine; cross-coupling; polymer-supported synthesis

\section{Introduction}

Palladium-catalyzed reactions that form carbon-carbon bonds, such as Suzuki-Miyaura, Mizoroki-Heck, Sonogashira, and Tsuji-Trost cross-coupling, are of considerable importance in organic chemistry [1-11]. These cross-coupling reactions are generally catalyzed with soluble palladium complexes in an organic solvent or in a mixture of water and an organic solvent [12-15]. However, there has been a continuing challenge with such catalyst systems regarding their efficient separation and recycling, which are important considerations for economic and ecological reasons.

Heterogeneous palladium-catalyst systems have been found to be highly effective for overcoming some of these problems [16-20]. Heterogeneous catalysts generally comprise an organic or inorganic solid support, a linkage moiety, and a catalytic center prepared from a transition metal and a 
phosphine-based ligand. Therefore, catalytic reactions are carried out under nitrogen to avoid oxidizing the phosphine ligands. We recently developed an amphiphilic polystyrene-poly(ethylene glycol) (PS-PEG) resin-supported terpyridine-metal complex and found it to be an effective catalyst for coupling reactions in water under heterogeneous and aerobic conditions with high recyclability [21,22]. As an extension of that study, we investigated this catalytic utility of the PS-PEG-terpyridine-palladium(II) complex for the cross-coupling reaction in aqueous solution, with the aim to achieve more environmentally friendly reaction media. We report herein the results of these investigations and demonstrate that the complex effectively catalyzes the cross-coupling reaction in water (Scheme 1). This catalytic system offers three benefits: (1) It produces neither organic solvent waste nor metal-contaminated waste; (2) It is unharmed by oxygen and moisture; (3) It thus enables the cross-coupling reaction to meet green chemical requirements; and (4) It enables the cross-coupling reaction with high level of safety.

Scheme 1. Preparation of PS-PEG resin-bound terpyridine palladium complex.
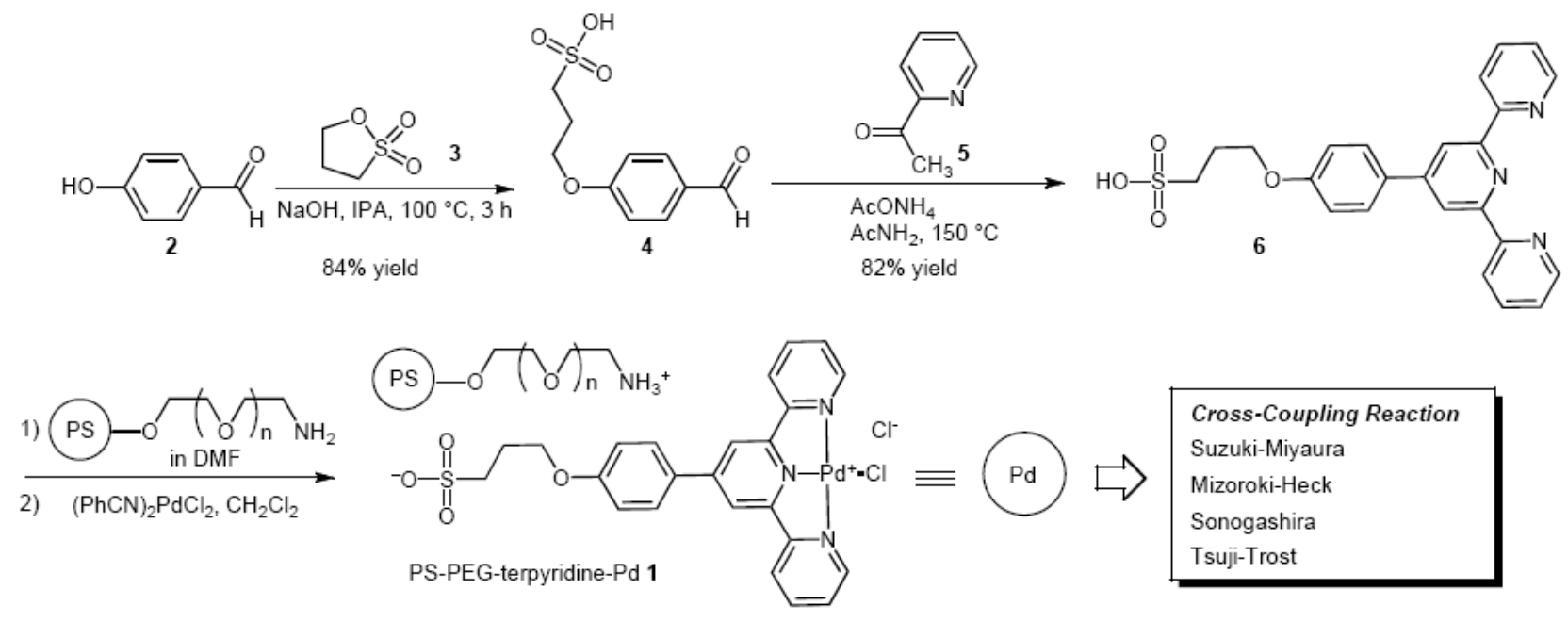

\section{Results and Discussion}

\subsection{Preparation of PS-PEG Resin-Bound Terpyridine Palladium Complex}

The amphiphilic PS-PEG resin-bound terpyridine ligand was prepared from $p$-hydroxybenzaldehyde (2), propane sultone (3), 2-acetylpyridine (5), and PS-PEG- $\mathrm{NH}_{2}$ resin, and the coordination of the synthesized polymeric terpyridine ligand with palladium species proceeded to give the PS-PEG resin-supported terpyridine palladium complex $\mathbf{1}$, which exhibited good catalytic activity for the cross-coupling reaction. Thus, the reaction of $p$-hydroxybenzaldehyde (2) with propane sultone (3) in isopropyl alcohol (IPA) at $100{ }^{\circ} \mathrm{C}$ for $3 \mathrm{~h}$ under alkaline conditions followed by treatment of the obtained 3-(4-formylphenoxy)propane-1-sulfonic acid (4) with 2-acetylpyridine (5) and $\mathrm{AcONH}_{4}$ in acetamide at $150{ }^{\circ} \mathrm{C}$ for $5 \mathrm{~h}$ gave terpyridine ligand 6 in a $68 \%$ yield in two steps. Immobilization of terpyridine ligand 6 onto an amphiphilic PS-PEG resin through ionic bonds to the sulfonate group was performed in DMF at room temperature for $1 \mathrm{~h}$ and the complexation of an amphiphilic PS-PEG resin-bound terpyridine ligand and $\mathrm{Pd}$ (II) occurred in $\mathrm{CH}_{2} \mathrm{Cl}_{2}$ to give PS-PEG-terpyridine-Pd(II) complex 1 as a brown solid [23-26]. 


\subsection{Suzuki-Miyaura Cross-Coupling Reaction Using Polymer-Supported Terpyridine Palladium Complex}

The scope of aryl halides and arylboronic acids was examined for the Suzuki-Miyaura cross-coupling reaction in water using the PS-PEG-terpyridine-Pd(II) complex $\mathbf{1}$ under aerobic conditions. Representative results are summarized in Table 1. PS-PEG resin-supported terpyridine palladium(II) complex $\mathbf{1}$ efficiently catalyzed the coupling of iodobenzene (7a) with phenylboronic acid (8a). Thus, the Suzuki-Miyaura cross-coupling reaction of 7a with 8a was carried out with $\mathrm{K}_{2} \mathrm{CO}_{3}$ ( 2 equiv.) in the presence of polymeric catalyst 1 ( $5 \mathrm{~mol} \% \mathrm{Pd})$ in water to give the biphenyl (9a) in a 93\% yield (Table 1, entry 1 ). The Suzuki-Miyaura cross-coupling reaction of phenylboronic acid (8a) with iodobenzene derivatives $\mathbf{7 b}-\mathbf{d}$ bearing electron donating and withdrawing substituents at their para-positions gave the 4-methylbiphenyl (9b), 4-methoxybiphenyl (9c), and 4-trifluoromethylbiphenyl (9d) in $82 \%, 92 \%$, and 93\% yields, respectively (Table 1, entries 2-4). The coupling reaction of metaand ortho-substituted iodobenzene derivatives 7e-g having meta-alkyl and ortho-alkyl groups took place with phenylboronic acid (8a) to give the 2-methylbiphenyl (9e), 3-methylbiphenyl (9f), and 1-phenylnaphthalene (9g) in 80\%, 93\%, and 98\% yields, respectively (Table 1, entries 5-7). The coupling reaction of bromoarenes $\mathbf{7 h}-\mathbf{k}$ with $\mathbf{8 a}$ was also performed to give the corresponding biaryls 9a-d in 74\%, 85\%, 69\%, and 82\% yields, respectively (Table 1, entries 8-11). The reactivity of several boronic acids $\mathbf{8 b}-\mathbf{g}$ was also examined under similar conditions to furnish the coupling products $\mathbf{9 b}$ and $\mathbf{9 e - i}$ in $91 \%, 87 \%, 90 \%, 92 \%, 99 \%$, and $96 \%$ yields, respectively (Table 1, entries 12-17).

Table 1. Suzuki-Miyaura coupling reaction using polymeric catalyst $\mathbf{1}$ in water $^{a, b}$.
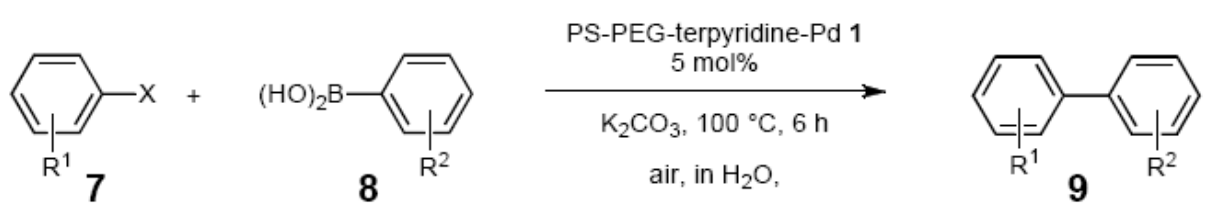

entry aryl halide (7)


Table 1. Cont.

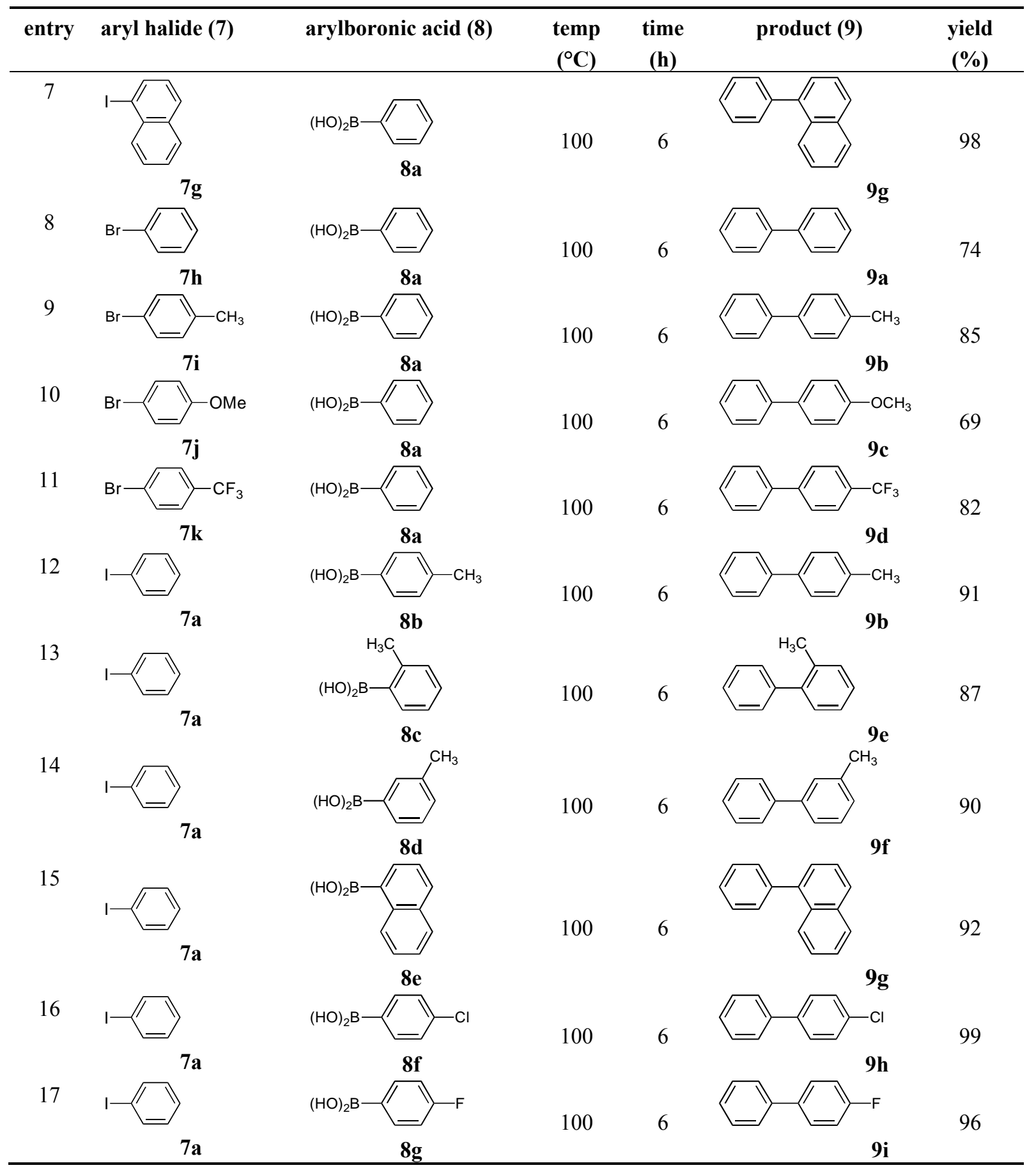

${ }^{a}$ All reactions were carried out with $\mathrm{ArX}(\mathbf{7} ; 0.4 \mathrm{mmol}), \mathrm{ArB}(\mathrm{OH})_{2}(\mathbf{8} ; 0.8 \mathrm{mmol})$, and $\mathrm{K}_{2} \mathrm{CO}_{3}(0.8 \mathrm{mmol})$ in the presence of the polymeric catalyst 1 in $3.0 \mathrm{~mL}$ of $\mathrm{H}_{2} \mathrm{O}$ at $100{ }^{\circ} \mathrm{C}$ for $6 \mathrm{~h}$ under aerobic conditions. ${ }^{\mathrm{b}}$ The $0.005 \mathrm{~mol} \%$ of polymeric catalyst $1(0.08 \mathrm{mg})$ was used, the reaction gave $74 \%$ yield of biphenyl $9 \mathrm{a}$ (TON 14800). ${ }^{\mathrm{c}}$ The iodobenzoic acid as water soluble substrate was used, the reaction afforded $59 \%$ yield of biphenyl-4-carboxylic acid $\mathbf{9 j}$.

\subsection{Mizoroki-Heck Reaction Using Polymer-Supported Terpyridine Palladium Complex}

The scope of suitable aryl halides and styrenes for the Mizoroki-Heck reaction in water using catalyst 1 was also examined (Table 2). The general process was to conduct the reaction of an aryl halide and a styrene with 1,8-diazabicyclo[5.4.0]undec-7-ene (DBU) (2 equiv.) in the presence of catalyst 1 ( $5 \mathrm{~mol} \% \mathrm{Pd}$ ) in water to afford the corresponding products. The results for various aryl halides and 
styrenes are as follows. For aryl halides $\mathbf{7 a}-\mathbf{g}$ in reaction with styrene 10a, iodobenzene (7a) gave 1,2-diphenylethene (11a) in a $90 \%$ yield (Table 2, entry 1); iodobenzene derivatives $\mathbf{7 b}$-d bearing electron donating and withdrawing substituents at their para-positions gave 1-methyl-4-styrylbenzene (11b), 1-methoxy-4-styrylbenzene (11c), and 1-trifluoromethyl-4-styrylbenzene (11d) in 78\%, 88\%, and $76 \%$ yields, respectively (Table 2, entries 2-4); in addition, meta- and ortho-substituted iodobenzene 7e-g having meta-methyl and ortho-methyl groups gave 1-methyl-2-styrylbenzene (11e), 1-methyl-3-styrylbenzene (11f), and 1-styrylnaphthalene (11g) in 60\%, 90\%, and 99\% yields, respectively (Table 2, entries 5-7). Bromoarenes $\mathbf{7 h}-\mathbf{k}$ in reaction with $\mathbf{1 0 a}$, carried out over longer reaction times (24 h), gave the corresponding stilbene derivatives $\mathbf{1 1 a - d}$ in $80 \%, 57 \%, 75 \%$, and $87 \%$ yields, respectively (Table 2, entries 9-12). Styrenes $\mathbf{1 0 b}$ and 10c under similar conditions gave 11b and $\mathbf{1 1 h}$ in $80 \%$ and $77 \%$ yields, respectively (Table 2, entries 13 and 14).

Table 2. Mizoroki-Heck reaction using polymeric catalyst $\mathbf{1}$ in water ${ }^{a, b, c}$.

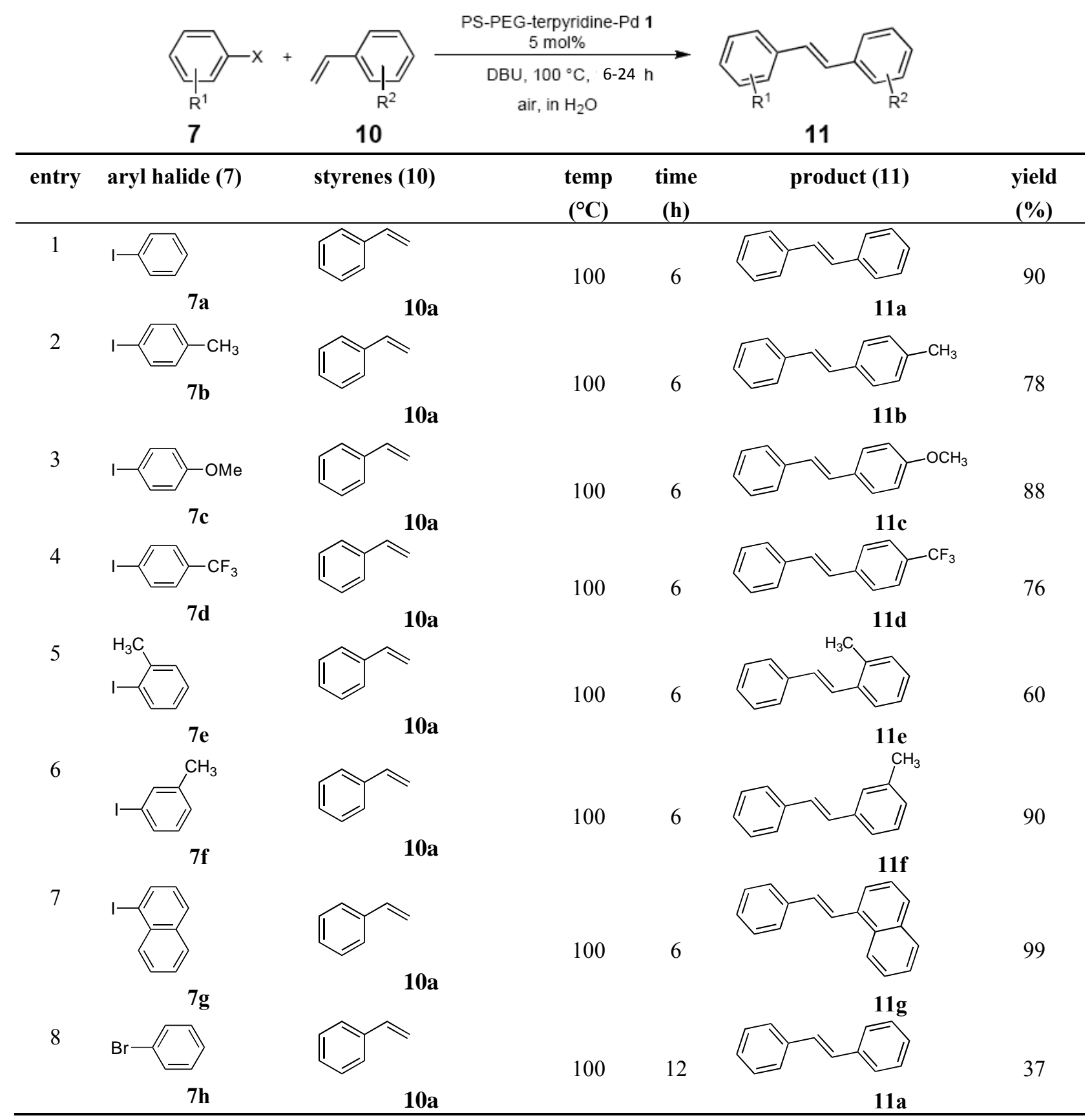


Table 2. Cont.

entry aryl halide (7) styrenes (10)

${ }^{a}$ All reactions were carried out with $\operatorname{ArX}(\mathbf{7} ; 0.4 \mathrm{mmol})$, styrenes $(\mathbf{1 0} ; 0.8 \mathrm{mmol})$, and DBU $(0.8 \mathrm{mmol})$ in the presence of the polymeric catalyst $\mathbf{1}$ in $3.0 \mathrm{~mL}$ of $\mathrm{H}_{2} \mathrm{O}$ at $100{ }^{\circ} \mathrm{C}$ for $12 \mathrm{~h}$ under aerobic conditions. ${ }^{\mathrm{b}}$ The $0.05 \mathrm{~mol} \%$ of polymeric catalyst $\mathbf{1}(0.8 \mathrm{mg}$ ) was used, the reaction gave $4 \%$ yield of stilbene $\mathbf{1 1 g}$ (TON 80 ).

\subsection{Sonogashira Coupling Reaction Using Polymer-Supported Terpyridine Palladium Complex}

The palladium-catalyzed coupling of an aryl halide with a terminal alkyne, the so-called Sonogashira reaction, is recognized as the most successful method for forming an $s p^{2}$ carbon-sp ${ }^{3}$ carbon bond. Since its discovery by Sonogashira and co-workers in 1975, a vast amount of research has been devoted to the synthetic application as well as the improvement of the reaction efficiency. Our continuing interest in the catalytic utility of PS-PEG-terpyridine-Pd(II) complex $\mathbf{1}$ led us to examine the Sonogashira coupling reaction in water with the PS-PEG-terpyridine-Pd(II) complex 1 under copper-free conditions. Thus, the copper-free Sonogashira coupling was examined with several haloarenes in the presence of catalyst $\mathbf{1}$ in water under aerobic conditions. The representative results are summarized in Table 3. The coupling of iodobenzene (7a) with phenylacetylene (12) took place smoothly in water at $60{ }^{\circ} \mathrm{C}$ in the presence of 3 equiv. of $\mathrm{Et}_{3} \mathrm{~N}$ and $5 \mathrm{~mol} \%$ palladium of the PS-PEG-terpyridine-Pd(II) complex 1 to give a $78 \%$ yield of diphenylacetylene (13a) (Table 3, entry 1). The Sonogashira coupling reaction of $p$-methyliodobenzene (7b) bearing electron donating groups at their para-positions gave the corresponding biarylacetylene 13b in a $65 \%$ yield (Table 3 , entry 2). $p$-(Trifluoromethyl)iodobenzene (7d) and $p$-(methoxycarbonyl)iodobenzene (7l) having electron deficient aromatic rings also underwent the Sonogashira coupling with phenylacetylene under similar conditions to afford the corresponding biarylacetylenes 13c and 13d in $82 \%$ and $69 \%$ yields, respectively (Table 3, entries 3 and 4). The coupling of meta- and ortho-substituted iodobenzenes $\mathbf{7 e - g , ~ m , ~ n ~ h a v i n g ~ m e t a - m e t h y l , ~ m e t a - c h l o r o , ~ o r t h o - m e t h y l , ~ a n d ~ o r t h o - c h l o r o ~ g r o u p s ~ t o o k ~ p l a c e ~ w i t h ~}$ phenylacetylene to give the corresponding products $13 \mathbf{e}-\mathbf{i}$ in $51 \%, 51 \%, 72 \%, 83 \%$, and $76 \%$ yields, respectively (Table 3, entries 5-9). However, Bromoarene $\mathbf{7 h}$ in reaction with $\mathbf{1 2}$, carried out over 
longer reaction times $(24 \mathrm{~h})$ and at a higher temperature $\left(100{ }^{\circ} \mathrm{C}\right)$, gave the corresponding stilbene 13a in a low yield (<5\% yield) (Table 3 , entry 11$)$.

Table 3. Sonogashira coupling reaction using polymeric catalyst 1 in water ${ }^{a, b}$.
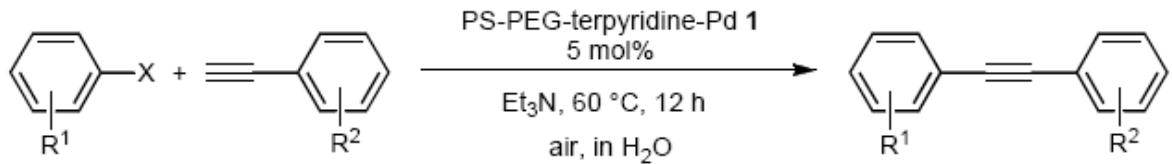

$7 \quad 12$

13

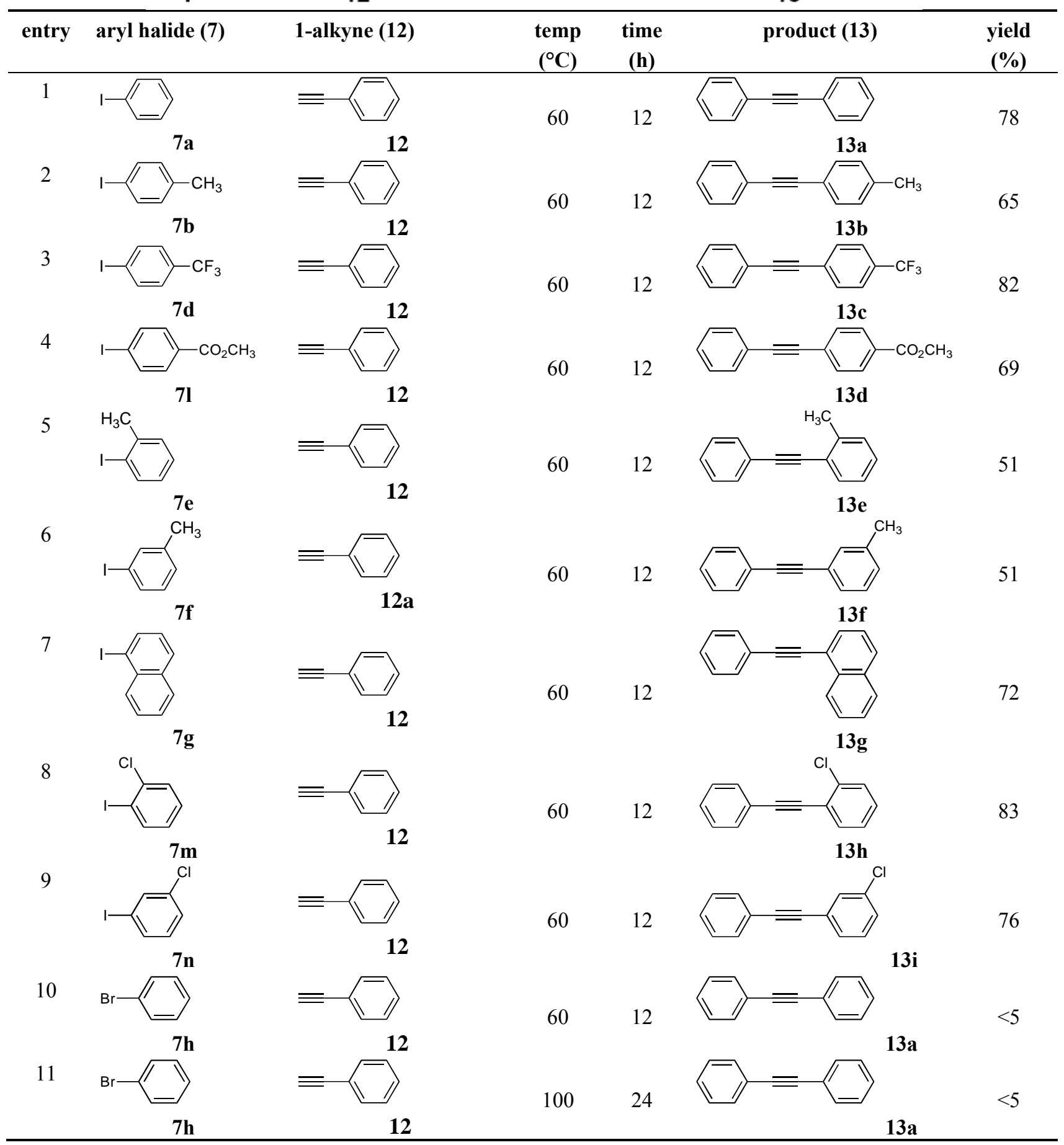

${ }^{a}$ All reactions were carried out with $\operatorname{ArX}(\mathbf{7} ; 0.4 \mathrm{mmol})$, phenyl acetylene $(\mathbf{1 2} ; 0.8 \mathrm{mmol})$, and $\mathrm{Et}_{3} \mathrm{~N}$ $(1.2 \mathrm{mmol})$ in the presence of the polymeric catalyst $1 \mathrm{in} 3.0 \mathrm{~mL}$ of $\mathrm{H}_{2} \mathrm{O}$ at $60{ }^{\circ} \mathrm{C}$ for $12 \mathrm{~h}$ under aerobic conditions. ${ }^{\mathrm{b}}$ The $0.005 \mathrm{~mol} \%$ of polymeric catalyst $\mathbf{1}(0.08 \mathrm{mg})$ was used, the reaction gave $28 \%$ yield of diphenyl acetylene 13a (TON 5600). 


\subsection{Tsuji-Trost Reaction Using Polymer-Supported Terpyridine Palladium Complex}

The palladium-catalyzed allyl esters via $\pi$-allylpalladium intermediates, the so-called Tusji-Trost reaction, are a powerful synthetic means for forming carbon-carbon as well as carbon-nitrogen bonds. While extensive research has been devoted to the $\pi$-allylic alkylation and amination, research on $\pi$-allylic azidation has been limited to isolated reports. We also describe herein our preliminary results demonstrating that allylic azidation of several allyl esters with sodium azide proceeded in water in the presence of a polymeric catalyst $\mathbf{1}$. Thus, the coupling of allyl acetate $\mathbf{1 4 a}$ an $\mathbf{1 4 b}$ with sodium azide 15 took place smoothly in water at $70{ }^{\circ} \mathrm{C}$ in the presence of $5 \mathrm{~mol} \%$ palladium of the PS-PEG-terpyridinePd(II) complex 1 to give the allyl azides $\mathbf{1 6 a}$ and $\mathbf{1 6 b}$ in $78 \%$ and $36 \%$ yields, respectively (Table 4, entries 1 and 2). The Tsuji-Trost reaction of 1-tolylallyl acetate (14c) bearing electron donating groups at their para-positions gave the 3-(p-tolyl)allyl azide (16c) in a 65\% yield (Table 4, entry 3). 1-(4-(trifluoromethyl)phenyl)allyl acetate (14d) having electron deficient aromatic rings also underwent the Tsuji-Trost reaction with sodium azide under similar conditions to afford the 3 -( $p$-trifluoromethylphenyl)allyl azide (16d) in an 18\% yield (Table 4, entry 4).

Table 4. Tsuji-Trost reaction using polymeric catalyst 1 in water ${ }^{a, b}$.

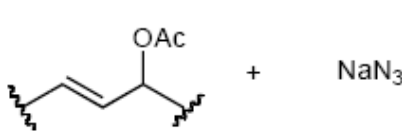

14

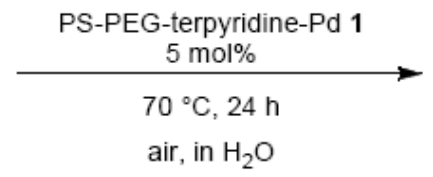

15

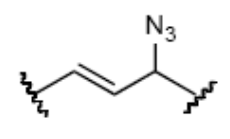

16

entry allyl acetate (14) nucleophile (15)

${ }^{a}$ All reactions were carried out with allyl acetate $(\mathbf{1 4} ; 0.4 \mathrm{mmol})$ and sodium azide $(\mathbf{1 5} ; 0.6 \mathrm{mmol})$ in the presence of the polymeric catalyst 1 in $3.0 \mathrm{~mL}$ of $\mathrm{H}_{2} \mathrm{O}$ at $70{ }^{\circ} \mathrm{C}$ for $24 \mathrm{~h}$ under aerobic conditions. ${ }^{\mathrm{b}}$ The $0.05 \mathrm{~mol} \%$ of polymeric catalyst $\mathbf{1}(0.8 \mathrm{mg})$ was used, the reaction gave $73 \%$ yield of azide $\mathbf{1 6 a}$ (TON 1460). 


\subsection{Recycling Experiments for Cross-Coupling Reaction in Water}

The recyclability of the PS-PEG-terpyridine-Pd(II) complex $\mathbf{1}$ was also tested for the Suzuki-Miyaura, Mizoroki-Heck, Sonogashira, and Tsuji-Trost coupling reaction, respectively. Thus, after the first reaction, which gave a good yield of the coupling product $9 \mathbf{a}, \mathbf{1 1 g}, \mathbf{1 3 h}$, and 16a (Table 1, entry 1 (93\% yield); Table 2, entry 7 (99\% yield); Table 3, entry 8 ( $83 \%$ yield); and Table 4, entry 1 ( $78 \%$ yield)), the catalyst beads were recovered by simple filtration and directly applied for the reaction under the similar conditions to afford 9a, 11g, 13a, and 16a in high yields (Scheme 2), during which palladium residue could hardly be detected by inductively coupled plasma atomic emission spectroscopy (ICP-AES) analysis (detection limit of $\mathrm{Pd}:<3 \mu \mathrm{g} / \mathrm{L}$ ) from aqueous nor organic filtrates, excluding the Mizoroki-Heck reaction (leaching of Pd: 5 ppm).

Scheme 2. Recycling experiments for cross-coupling reaction in water.

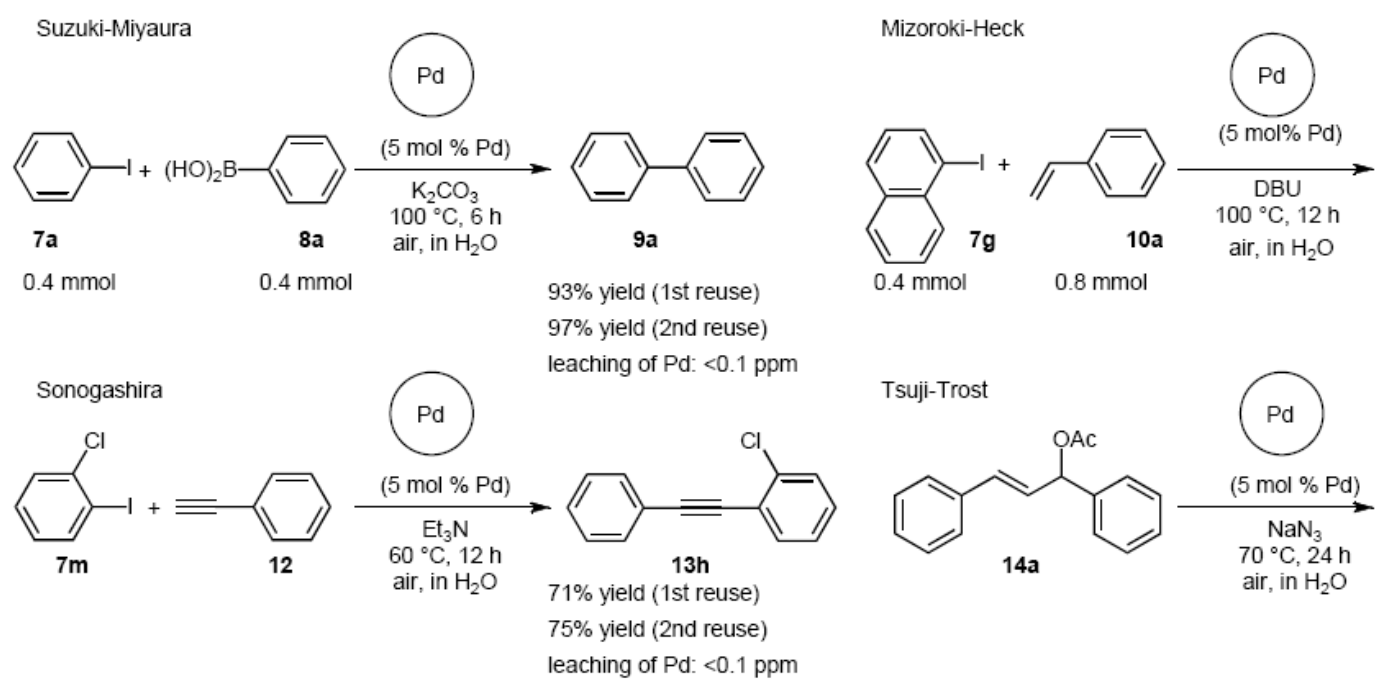

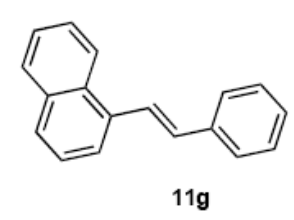

$97 \%$ yield (1st reuse) $99 \%$ yield ( 2 nd reuse) leaching of Pd: 5 ppm

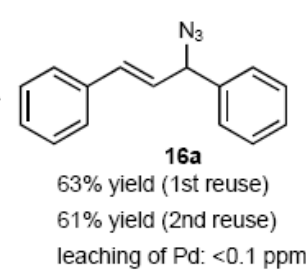

\section{Experimental Section}

\subsection{General Methods}

All manipulations were carried out under aerobic conditions. Water was deionized with a Millipore MilliQ gradient A10 as Milli-Q grade. NMR spectra were recorded on a BURKER AVANCE spectrometer (500 MHz for ${ }^{1} \mathrm{H}$ and $125 \mathrm{MHz}$ for ${ }^{13} \mathrm{C}$ ) and NMR spectra were recorded on a BURKER AVANCE spectrometer (400 MHz for ${ }^{1} \mathrm{H}$ and $100 \mathrm{MHz}$ for ${ }^{13} \mathrm{C}$ ), and HITACHI R1900 spectrometer (90 MHz for ${ }^{1} \mathrm{H}$ and $22 \mathrm{MHz}$ for ${ }^{13} \mathrm{C}$ ). ${ }^{1} \mathrm{H}$ and ${ }^{13} \mathrm{C}$ NMR spectra were recorded in $\mathrm{CDCl}_{3}$ or dimethyl sulfoxide- $d_{6}$ (DMSO- $d_{6}$ ) at $25{ }^{\circ} \mathrm{C}$. Chemical shifts of ${ }^{13} \mathrm{C}$ NMR were given relative to $\mathrm{CDCl}_{3}$ and DMSO- $d_{6}$ as an internal standard ( $\delta 77.0 \mathrm{ppm}$ and $\left.39.7 \mathrm{ppm}\right)$. Mass spectral data were measured on a JEOL JMS-T100GCv MS detector (GC-MS) and a JEOL JMS-T100LP MS detector (LC-MS); the abbreviation ' $\mathrm{bp}$ ' is used to denote the base peak. GC analysis was performed on a Shimadzu GC-2014. IR analysis was performed on a JASCO FTIR-410. ICP-AES spectral data were measured on a Shimadzu ICPE-9000. 


\subsection{Materials}

PS-PEG-supported terpyridine-palladium complex (PS-PEG-terpyridine-Pd) $\mathbf{1}$ was prepared from PS-PEG amino-resin (Tenta Gel S NH 2 , average diameter $90 \mathrm{~mm}, 1 \%$ divinylbenzene cross-linked, loading value of amino residue $0.31 \mathrm{mmol} / \mathrm{g}$; purchased from Rapp Polymer), polymeric terpyridine ligand, and $\left(\mathrm{C}_{6} \mathrm{H}_{5} \mathrm{CN}\right)_{2} \mathrm{PdCl}_{2}$ according to the reported procedures [22]. The loading level of $\mathrm{Pd}$ in polymeric catalyst 1 was $0.25 \mathrm{mmol} / \mathrm{g}$.

\subsection{Suzuki-Miyaura Cross-Coupling Reaction}

To a mixture of the polymeric catalyst $1(80 \mathrm{mg}, 0.020 \mathrm{mmol}), \mathrm{K}_{2} \mathrm{CO}_{3}(110 \mathrm{mg}, 0.8 \mathrm{mmol})$, and iodobenzene $(\mathbf{7 a} ; 81 \mathrm{mg}, 0.4 \mathrm{mmol})$ in $\mathrm{H}_{2} \mathrm{O}(3.0 \mathrm{~mL})$ was added phenyl boronic acid $(\mathbf{8 a} ; 97 \mathrm{mg}$, $0.8 \mathrm{mmol}$ ). The reaction mixture was shaken at $100{ }^{\circ} \mathrm{C}$ for $6 \mathrm{~h}$ and filtered. The recovered resin beads were rinsed with $\mathrm{H}_{2} \mathrm{O}$ and extracted three times with EtOAc $(6 \mathrm{~mL})$. The EtOAc layer was separated and the aqueous layer was extracted with EtOAc $(3 \mathrm{~mL})$. The combined EtOAc extracts were washed with brine $(2 \mathrm{~mL})$, dried over $\mathrm{MgSO}_{4}$, and concentrated in vacuo. The resulting residue was chromatographed on silica gel (hexane) to give $57 \mathrm{mg}$ (93\% yield) of biphenyl (9a).

\subsection{Spectral and Analytical Data for the Biaryls}

\subsection{1. biphenyl (9a)}

${ }^{1} \mathrm{H}$ NMR $\left(\mathrm{CDCl}_{3}\right) \delta 7.60-7.57(\mathrm{~m}, 2 \mathrm{H}), 7.45-7.41(\mathrm{~m}, 4 \mathrm{H}), 7.35-7.31(\mathrm{~m}, 4 \mathrm{H}):{ }^{13} \mathrm{C} \mathrm{NMR}\left(\mathrm{CDCl}_{3}\right)$ $\delta 141.2$ (2 C), 128.7 (4 C), 127.2 (4 C), 127.1 (2 C). MS (EI): $m / z(r e l \%) 154$ (bp, M ${ }^{+}$), 153 (39), 77 (6). IR (ATR): $\left(\mathrm{cm}^{-1}\right)$ v 3061, 1475.

CAS registry number: 92-52-4.

\subsubsection{4-methylbiphenyl (9b)}

${ }^{1} \mathrm{H}$ NMR $\left(\mathrm{CDCl}_{3}\right) \delta$ 7.60-7.55 (m, $\left.2 \mathrm{H}\right), 7.50-7.47(\mathrm{~m}, 2 \mathrm{H}), 7.43-7.39(\mathrm{~m}, 2 \mathrm{H}), 7.33-7.29$ $(\mathrm{m}, 1 \mathrm{H}), 7.25-7.20(\mathrm{~m}, 2 \mathrm{H}), 2.38(\mathrm{~s}, 3 \mathrm{H}):{ }^{13} \mathrm{C} \mathrm{NMR}\left(\mathrm{CDCl}_{3}\right) \delta 141.1,138.3,136.9$, $129.4(2 \mathrm{C})$, 128.7 (2 C), 128.6 (2 C), 126.9 (2 C), 126.9, 21.0. MS (EI): $m / z(r e l \%) 168$ (bp, $\mathrm{M}^{+}$), 167 (69), 165 (24), 153 (13), 83 (9). IR (ATR): $\left(\mathrm{cm}^{-1}\right)$ v 3026, 1486.

CAS registry number: 644-08-6.

\subsubsection{4-methoxybiphenyl (9c)}

${ }^{1} \mathrm{H} \mathrm{NMR}\left(\mathrm{CDCl}_{3}\right) \delta 7.52(\mathrm{t}, J=8.6 \mathrm{~Hz}, 4 \mathrm{H}), 7.39(\mathrm{t}, J=7.5 \mathrm{~Hz}, 2 \mathrm{H}), 7.28(\mathrm{t}, J=7.5 \mathrm{~Hz}, 1 \mathrm{H})$, $6.96(\mathrm{~d}, J=8.6 \mathrm{~Hz}, 2 \mathrm{H}), 3.82(\mathrm{~s}, 3 \mathrm{H}):{ }^{13} \mathrm{C} \mathrm{NMR}\left(\mathrm{CDCl}_{3}\right) \delta 159.1,140.7,133.7,128.6(2 \mathrm{C}), 128.0$ (2 C), 126.6 (2 C), 126.6 , 114.1 (2 C), 55.2. MS (EI): $m / z$ (rel\%) 184 (bp, $\mathrm{M}^{+}$), 169 (53), 141 (46), 115 (29). IR (ATR): $\left(\mathrm{cm}^{-1}\right) \vee 3065,1603$.

CAS registry number: 613-37-6. 


\subsubsection{4-trifluoromethylbiphenyl (9d)}

${ }^{1} \mathrm{H}$ NMR $\left(\mathrm{CDCl}_{3}\right) \delta 7.67(\mathrm{br}, 4 \mathrm{H}), 7.59-7.57(\mathrm{~m}, 2 \mathrm{H}), 7.47-7.43(\mathrm{~m}, 2 \mathrm{H}), 7.40-7.37(\mathrm{~m}, 1 \mathrm{H}):{ }^{13} \mathrm{C}$ $\operatorname{NMR}\left(\mathrm{CDCl}_{3}\right) \delta 144.7,139.7,129.3(\mathrm{q}, J=32.5 \mathrm{~Hz}, 1 \mathrm{C}), 128.9(2 \mathrm{C}), 128.1(2 \mathrm{C}), 127.5,127.2(2 \mathrm{C})$, 125.6 (q, $J=3.7 \mathrm{~Hz}, 2 \mathrm{C}), 124.4$ (q, $J=271.8 \mathrm{~Hz}, 1 \mathrm{C}) . \mathrm{MS}(\mathrm{EI}): m / z(\mathrm{rel} \%) 222\left(\mathrm{bp}, \mathrm{M}^{+}\right), 203(8), 153$ (17). HRMS (EI): calcd for $\mathrm{C}_{13} \mathrm{H}_{9} \mathrm{~F}_{3} 222.0656$, found 222.0655. IR (ATR): $\left(\mathrm{cm}^{-1}\right) v 3034,1613,1111$.

\subsubsection{2-methylbiphenyl (9e)}

${ }^{1} \mathrm{H}$ NMR $\left(\mathrm{CDCl}_{3}\right) \delta$ 7.57-7.38 (m, $\left.2 \mathrm{H}\right), 7.35-7.30(\mathrm{~m}, 3 \mathrm{H}), 7.24-7.20(\mathrm{~m}, 4 \mathrm{H}), 2.26(\mathrm{~s}, 3 \mathrm{H}):{ }^{13} \mathrm{C}$ NMR $\left(\mathrm{CDCl}_{3}\right) \delta 141.9,141.9,135.3,130.2,129.7,129.1,128.7,128.0,127.2,127.1,126.7,125.7,20.4$. MS (EI): $m / z(r e l \%) 168\left(\mathrm{bp}, \mathrm{M}^{+}\right), 165$ (38), 152 (30). IR (ATR): $\left(\mathrm{cm}^{-1}\right) v 3061,1598,1478,1073$.

CAS registry number: 643-58-3.

\subsubsection{3-methylbiphenyl (9f)}

${ }^{1} \mathrm{H}$ NMR $\left(\mathrm{CDCl}_{3}\right) \delta$ 7.58-7.56 (m, $\left.2 \mathrm{H}\right), 7.42-7.37(\mathrm{~m}, 4 \mathrm{H}), 7.32-7.29(\mathrm{~m}, 2 \mathrm{H}), 7.15-7.13$ $(\mathrm{m}, 1 \mathrm{H}), 2.40(\mathrm{~s}, 3 \mathrm{H}):{ }^{13} \mathrm{C} \mathrm{NMR}\left(\mathrm{CDCl}_{3}\right) \delta 141.3,141.2,138.2,128.6(2 \mathrm{C}), 127.96(2 \mathrm{C}), 127.16(2$ C), 124.26 (2 C), 21.4. MS (EI): $m / z(\operatorname{rel} \%) 168$ (bp, $\left.\mathrm{M}^{+}\right), 167$ (58), 165 (24), 115 (6), 91 (5). IR (ATR): $\left(\mathrm{cm}^{-1}\right)$ v 3057, 1600, 1481, 1027.

CAS registry number: 643-93-6.

\subsubsection{1-phenylnaphthalene $(9 \mathrm{~g})$}

${ }^{1} \mathrm{H} \mathrm{NMR}\left(\mathrm{CDCl}_{3}\right) \delta 7.88(\mathrm{t}, J=9.0 \mathrm{~Hz}, 2 \mathrm{H}), 7.82(\mathrm{~d}, J=8.2 \mathrm{~Hz}, 1 \mathrm{H}), 7.56-7.33(\mathrm{~m}, 9 \mathrm{H}):{ }^{13} \mathrm{C}$ NMR $\left(\mathrm{CDCl}_{3}\right) \delta 140.7,140.2,133.7,131.6,130.0$ (2 C), 128.7, 128.2 (2 C), 127.6, 127.1, 127.1, 126.8, 125.9, 125.7, 125.3. MS (EI): $m / z$ (rel\%) 204 (bp, $\left.\mathrm{M}^{+}\right), 203$ (99), 201 (12), 101 (23). IR (ATR): $\left(\mathrm{cm}^{-1}\right)$ v 3055, 1590, 1493, 1019.

CAS registry number: 605-02-7.

\subsubsection{4-fluorobiphenyl (9i)}

${ }^{1} \mathrm{H}$ NMR $\left(\mathrm{CDCl}_{3}\right) \delta$ 7.72-7.50 (m, $\left.4 \mathrm{H}\right), 7.43-7.41(\mathrm{~m}, 2 \mathrm{H}), 7.39-7.30(\mathrm{~m}, 1 \mathrm{H}), 7.17-7.08$ $(\mathrm{m}, 2 \mathrm{H}):{ }^{13} \mathrm{C} \mathrm{NMR}\left(\mathrm{CDCl}_{3}\right) \delta 162.4(\mathrm{~d}, J=246.3 \mathrm{~Hz}, 1 \mathrm{C}), 140.2,137.3(\mathrm{~d}, J=3.2 \mathrm{~Hz}, 2 \mathrm{C}), 128.7$, 128.6, 128.5, 127.2, 126.9 (2 C), 115.5 (d, $J=21.4 \mathrm{~Hz}, 2 \mathrm{C})$. MS (EI): $m / z(\mathrm{rel} \%) 172\left(\mathrm{bp}, \mathrm{M}^{+}\right), 171$ (47), 85 (9). IR (ATR): $\left(\mathrm{cm}^{-1}\right) v 3042,1596,1482,1233$.

CAS registry number: $324-74-3$.

\subsubsection{4-chlorobiphenyl (9h)}

${ }^{1} \mathrm{H}$ NMR $\left(\mathrm{CDCl}_{3}\right) \delta$ 7.57-7.49 (m, $\left.4 \mathrm{H}\right), 7.46-7.34(\mathrm{~m}, 5 \mathrm{H}):{ }^{13} \mathrm{C} \mathrm{NMR}\left(\mathrm{CDCl}_{3}\right) \delta 139.9,139.6$, 133.3, 128.8 (4C), 128.3 (2C), 127.5, 126.9 (2C). MS (EI): $m / z(r e l \%) 188$ (bp, $\left.\mathrm{M}^{+}\right), 153$ (23), 151 (12), 94 (4), 51 (4). IR (ATR): $\left(\mathrm{cm}^{-1}\right) v$ 3065, 3038, 1475, 1096.

CAS registry number: 2051-62-9. 


\subsubsection{0. biphenyl-4-carboxylic acid (9j)}

${ }^{1} \mathrm{H}$ NMR $\left(\mathrm{CDCl}_{3}\right) \delta 12.5(\mathrm{br}, 1 \mathrm{H}), 8.09(\mathrm{~d}, \mathrm{~J}=8.5 \mathrm{~Hz}, 1 \mathrm{H}), 7.72-7.65(\mathrm{~m}, 4 \mathrm{H}), 7.39-7.29(\mathrm{~m}, 4$ $\mathrm{H}):{ }^{13} \mathrm{C} \mathrm{NMR}\left(\mathrm{CDCl}_{3}\right) \delta 146.7,141.3,134.6,131.2,130.0,129.1$ (2 C), 128.5 (2 C), $128.1(2 \mathrm{C}), 127.9$ (2 C). MS (ESI): $221.0\left(\mathrm{M}+\mathrm{Na}^{+}\right)$. IR (ATR): $\left(\mathrm{cm}^{-1}\right) v 3446,3080,1677,1602$.

CAS registry number: 92-92-2.

\subsection{Mizoroki-Heck Reaction}

The general procedure to obtain Mizoroki-Heck reaction products $\mathbf{1 1 a}-\mathbf{h}$ is as described here for 11a. Styrene $(\mathbf{1 1 a} ; 83 \mathrm{mg}, 0.8 \mathrm{mmol})$ was added to a mixture of polymeric catalyst $(\mathbf{1} ; 80 \mathrm{mg}$, $0.020 \mathrm{mmol})$, base DBU $(121 \mathrm{mg}, 0.8 \mathrm{mmol})$, and iodobenzene $(7 \mathbf{a} ; 81 \mathrm{mg}, 0.4 \mathrm{mmol})$ in $\mathrm{H}_{2} \mathrm{O}$ $(3.0 \mathrm{~mL})$. The reaction mixture was shaken at $100{ }^{\circ} \mathrm{C}$ for $12 \mathrm{~h}$ and then filtered. The recovered resin beads were rinsed with $\mathrm{H}_{2} \mathrm{O}$ and extracted three times with EtOAc $(6 \mathrm{~mL})$. The EtOAc layer was separated and the aqueous layer was extracted with EtOAc $(3 \mathrm{~mL})$. The combined EtOAc extracts were washed with brine $(2 \mathrm{~mL})$, dried over $\mathrm{MgSO}_{4}$, and concentrated in vacuo. The resulting residue was chromatographed on silica gel (hexane) to give $64.8 \mathrm{mg}$ (90\% yield) of (E)-1,2-diphenylethene (11a).

\subsection{Spectral and Analytical Data for the Stilbenes}

3.6.1. (E)-1,2-diphenylethene (11a)

${ }^{1} \mathrm{H}\left(\mathrm{CDCl}_{3}\right) \delta 7.57-7.39(\mathrm{~m}, 4 \mathrm{H}), 7.36-7.21(\mathrm{~m}, 6 \mathrm{H}), 7.14(\mathrm{~s}, 2 \mathrm{H}):{ }^{13} \mathrm{C}\left(\mathrm{CDCl}_{3}\right) \delta 137.5(2 \mathrm{C})$, 128.8 (2 C), 128.7 (4 C), 127.6 (2 C), 126.6 (2 C). MS (EI): m/z (rel\%) 180 (bp, M ${ }^{+}$), 179 (96), 165 (44). IR (ATR): $\left(\mathrm{cm}^{-1}\right)$ v 2922, 1737, 1450.

CAS registry number: 103-30-0.

3.6.2. (E)-1-methyl-4-styrylbenzene (11b)

${ }^{1} \mathrm{H} \mathrm{NMR}\left(\mathrm{CDCl}_{3}\right) \delta$ 7.53-7.08 (m, $\left.9 \mathrm{H}\right), 7.04(\mathrm{~s}, 2 \mathrm{H}), 2.33(\mathrm{~s}, 3 \mathrm{H}):{ }^{13} \mathrm{C} \mathrm{NMR}\left(\mathrm{CDCl}_{3}\right) \delta 137.7$, 137.5, 134.8, 129.4 (2 C), 128.8, 128.6 (2 C), 127.9, 127.4, 126.5 (2 C), 126.4 (2 C), 21.0. MS (EI): $\mathrm{m} / \mathrm{z}(\mathrm{rel} \%) 194\left(98, \mathrm{M}^{+}\right), 193$ (23), 179 (bp). IR (ATR): (cm $\left.{ }^{-1}\right) v$ 3020, 1592, 1508, 1448.

CAS registry number: 4714-21-0.

\subsection{3. (E)-1-methoxy-4-styrylbenzene (11c)}

${ }^{1} \mathrm{H} \mathrm{NMR}\left(\mathrm{CDCl}_{3}\right) \delta$ 7.50-7.20 (m, $\left.7 \mathrm{H}\right), 7.00-6.81(\mathrm{~m}, 4 \mathrm{H}), 3.79(\mathrm{~s}, 3 \mathrm{H}):{ }^{13} \mathrm{C} \mathrm{NMR}\left(\mathrm{CDCl}_{3}\right) \delta$ 159.2, 137.8, 128.6 (2 C), 128.4, 127.7 (2 C), 127.2 (2 C), 126.8, 126.3 (2 C), 114.3 (2 C), 55.2. MS (EI): $m / z(\mathrm{rel} \%) 210$ (bp, M $\mathrm{M}^{+}$), 195 (20), 167 (31), 165 (32). IR (ATR): (cm $\left.{ }^{-1}\right) v 2935,1599,1509$.

CAS registry number: $1142-15-0$. 
3.6.4. (E)-1-trifluoromethyl-4-styrylbenzene (11d)

${ }^{1} \mathrm{H}$ NMR $\left(\mathrm{CDCl}_{3}\right) \delta$ 7.55-7.29 (m, $\left.9 \mathrm{H}\right), 7.11-7.10(\mathrm{~m}, 2 \mathrm{H}):{ }^{13} \mathrm{C} \mathrm{NMR}\left(\mathrm{CDCl}_{3}\right) \delta 140.8,131.9$, 129.2 (q, $J=32.3 \mathrm{~Hz}, 1 \mathrm{C}), 128.7$ (2 C), 128.3 (2 C), 127.1, 126.9 (2 C), 126.5 (2 C), 125.6 (q, $J=3.9 \mathrm{~Hz}, 2 \mathrm{C}), 124.2$ (q, $J=271.5 \mathrm{~Hz}, 1 \mathrm{C}) . \mathrm{MS}(\mathrm{EI}): \mathrm{m} / z(\mathrm{rel} \%) 248$ (bp, $\left.\mathrm{M}^{+}\right), 179$ (74), 178 (62). HRMS (EI): calcd for $\mathrm{C}_{15} \mathrm{H}_{11} \mathrm{~F}_{3}$ 248.0812, found 248.0818. IR (ATR): (cm ${ }^{-1}$ ) v 2923, 1611, 1132.

3.6.5. (E)-1-methyl-2-styrylbenzene (11e)

${ }^{1} \mathrm{H} \mathrm{NMR}\left(\mathrm{CDCl}_{3}\right) \delta$ 7.56-7.11 (m, $\left.11 \mathrm{H}\right), 2.41(\mathrm{~s}, 3 \mathrm{H}):{ }^{13} \mathrm{C} \mathrm{NMR}\left(\mathrm{CDCl}_{3}\right) \delta 130.4,130.1,128.6$ (4 C), 127.5 (2 C), 126.7, 126.5, 126.2 (2 C), 125.5, 19.6. MS (EI): $m / z$ (rel\%) 194 (82, $\mathrm{M}^{+}$), 179 (bp), 115 (22). IR (ATR): $\left(\mathrm{cm}^{-1}\right)$ v 3023, 1494.

CAS registry number: 74685-42-0.

3.6.6. (E)-1-methyl-3-styrylbenzene (11f)

${ }^{1} \mathrm{H} \mathrm{NMR}\left(\mathrm{CDCl}_{3}\right) \delta 7.53-7.11(\mathrm{~m}, 9 \mathrm{H}), 7.05(\mathrm{~s}, 2 \mathrm{H}), 2.34(\mathrm{~s}, 3 \mathrm{H}):{ }^{13} \mathrm{C} \mathrm{NMR}\left(\mathrm{CDCl}_{3}\right) \delta 138.2$, 137.6, 137.5, 129.0, 128.6 (3 C), 128.6, 128.4, 127.5, 127.3, 126.5 (2 C), 123.8, 21.2. MS (EI): $\mathrm{m} / \mathrm{z}$ (rel\%) 194 (93, M+), 193 (28), 179 (bp). IR (ATR): $\left(\mathrm{cm}^{-1}\right)$ v 3022, 1598, 1493, 1448, 964.

CAS registry number: $14064-48-3$.

3.6.7. (E)-1-styrylnaphthalene (11g)

${ }^{1} \mathrm{H} \mathrm{NMR}\left(\mathrm{CDCl}_{3}\right) \delta 8.24-7.16(\mathrm{~m}, 14 \mathrm{H}):{ }^{13} \mathrm{C} \mathrm{NMR}\left(\mathrm{CDCl}_{3}\right) \delta 137.5,135.1,133.9,132.2,131.9$, 128.8 (2 C), 128.6, 128.0, 127.7, 126.7 (2 C), 126.0, 125.9, 125.9, 125.7, 123.8, 123.7. MS (EI): $\mathrm{m} / \mathrm{z}$ (rel\%) 230 (99, M+), 229 (bp), 228 (31), 152 (25). IR (ATR): $\left(\mathrm{cm}^{-1}\right) v$ 3055, 3022, 1493, 957.

CAS registry number: 2043-00-7.

3.6.8. (E)-1-chloro-4-styrylbenzene (11h)

${ }^{1} \mathrm{H} \mathrm{NMR}\left(\mathrm{CDCl}_{3}\right) \delta$ 7.52-7.26 (m, $\left.9 \mathrm{H}\right), 7.01(\mathrm{~s}, 2 \mathrm{H}):{ }^{13} \mathrm{C} \mathrm{NMR}\left(\mathrm{CDCl}_{3}\right) \delta 136.9,135.7,133.1$, 129.2, 128.7 (2 C), 128.6 (2 C), 127.8, 127.6 (2 C), 127.2, 126.5 (2 C). MS (EI): $\mathrm{m} / \mathrm{z}$ (rel\%) 214 (85, $\left.\mathrm{M}^{+}\right), 179$ (99), 89 (27), 76 (23). IR (ATR): $\left(\mathrm{cm}^{-1}\right) v$ 3021, 2923, 1488, 1087.

CAS registry number: 4714-23-2.

\subsection{Sonogashira Coupling Reaction}

The general procedure to obtain Sonogashira coupling reaction products $\mathbf{1 3 a}-\mathbf{g}$ is as described here for 13a. phenylacetylene $(\mathbf{1 2} ; 81.7 \mathrm{mg}, 0.8 \mathrm{mmol})$ was added to a mixture of polymeric catalyst $(\mathbf{1}$; $80 \mathrm{mg}, 0.024 \mathrm{mmol})$, base $\mathrm{Et}_{3} \mathrm{~N}(121 \mathrm{mg}, 1.2 \mathrm{mmol})$, and iodobenzene (7a; $\left.81.6 \mathrm{mg}, 0.4 \mathrm{mmol}\right)$ in $\mathrm{H}_{2} \mathrm{O}$ $(3.0 \mathrm{~mL})$. The reaction mixture was shaken at $60{ }^{\circ} \mathrm{C}$ for $12 \mathrm{~h}$ and then filtered. The recovered resin beads were rinsed with $\mathrm{H}_{2} \mathrm{O}$ and extracted three times with EtOAc $(6 \mathrm{~mL})$. The EtOAc layer was 
separated and the aqueous layer was extracted with EtOAc $(3 \mathrm{~mL})$. The combined EtOAc extracts were washed with brine $(2 \mathrm{~mL})$, dried over $\mathrm{MgSO}_{4}$, and concentrated in vacuo. The resulting residue was chromatographed on silica gel (hexane/AcOEt:100/1) to give $55.3 \mathrm{mg}$ (77\% yield) of diphenylacetylene (13a).

\subsection{Spectral and Analytical Data for the Biarylacetylenes}

3.8.1. Diphenylacetylene (13a)

${ }^{1} \mathrm{H}$ NMR $\left(\mathrm{CDCl}_{3}\right) \delta$ 7.55-7.51 (m, $\left.4 \mathrm{H}\right), 7.36-7.29(\mathrm{~m}, 6 \mathrm{H}):{ }^{13} \mathrm{C} \mathrm{NMR}\left(\mathrm{CDCl}_{3}\right) \delta 131.5,128.3$, 128.2, 123.2, 89.4. MS (EI): $\mathrm{m} / z$ (rel\%) 178 (bp, $\left.\mathrm{M}^{+}\right), 152$ (24). IR (ATR): (cm $\left.{ }^{-1}\right) v 3062,1598,1491$.

CAS registry number: 64666-02-0.

3.8.2. 1-Phenly-2-(p-tolyl)acetylene (13b)

${ }^{1} \mathrm{H}$ NMR $\left(\mathrm{CDCl}_{3}\right) \delta 7.52(\mathrm{dd}, J=8.1,1.9 \mathrm{~Hz}, 2 \mathrm{H}), 7.43(\mathrm{~d}, J=8.1 \mathrm{~Hz}, 2 \mathrm{H}), 7.38-7.30(\mathrm{~m}, 3 \mathrm{H})$, $7.15(\mathrm{~d}, J=7.8,2 \mathrm{H}), 2.36(\mathrm{~s}, 3 \mathrm{H}):{ }^{13} \mathrm{C} \mathrm{NMR}\left(\mathrm{CDCl}_{3}\right) \delta 138.3,134.8,131.5,131.4,129.1,128.3$, 128.0, 127.7, 120.1, 89.5, 21.5. MS (EI): $\mathrm{m} / \mathrm{z}(\mathrm{rel} \%) 192$ (bp, $\left.\mathrm{M}^{+}\right), 165$ (27), 39 (21). IR (ATR): $\left(\mathrm{cm}^{-1}\right)$ $v$ 3052, 3029, 2215, 1594, 1509, 1441, 1380.

CAS registry number: 185817-85-0.

3.8.3. 1-Phenyl-2-( $p$-trifluoromethylphenyl)acetylene (13c)

${ }^{1} \mathrm{H}$ NMR $\left(\mathrm{CDCl}_{3}\right) \delta 7.62(\mathrm{dd}, J=14.1,8.2 \mathrm{~Hz}, 4 \mathrm{H}), 7.56-7.54(\mathrm{~m}, 2 \mathrm{H}), 7.37(\mathrm{~m}, 3 \mathrm{H}):{ }^{13} \mathrm{C} \mathrm{NMR}$ $\left(\mathrm{CDCl}_{3}\right) \delta 132.5,131.8,131.7,129.8(\mathrm{q}, J=33.1 \mathrm{~Hz}, 1 \mathrm{C}), 128.8,128.4,126.4(\mathrm{q}, J=272 \mathrm{~Hz}, 1 \mathrm{C})$, 125.2 (q, $J=272 \mathrm{~Hz}, 1 \mathrm{C}), 122.5,91.7,87.9 . \mathrm{MS}(\mathrm{EI}): m / z(\operatorname{rel} \%) 246$ (73, $\left.\mathrm{M}^{+}\right), 176$ (43), 98 (bp), 75 (49), 51 (46). IR (ATR): $\left(\mathrm{cm}^{-1}\right) v 3080,2219,1508$.

CAS registry number: $370-99-0$.

3.8.4. 1-(p-Methoxycarbonylphenyl)-2-phenylacetylene (13d)

${ }^{1} \mathrm{H}$ NMR $\left(\mathrm{CDCl}_{3}\right) \delta 8.02(\mathrm{~d}, J=8.5 \mathrm{~Hz}, 2 \mathrm{H}), 7.59(\mathrm{~d}, J=8.5 \mathrm{~Hz}, 2 \mathrm{H}), 7.57-7.53(\mathrm{~m}, 2 \mathrm{H})$, 7.38-7.36 (m, $3 \mathrm{H}), 3.93$ (s, $3 \mathrm{H}):{ }^{13} \mathrm{C} \mathrm{NMR}\left(\mathrm{CDCl}_{3}\right) \delta 166.5,131.7,131.4,129.5,129.4,128.7,128.4$, 127.9, 122.6, 92.4, 88.6, 52.9. MS (EI): $m / z$ (rel\%) 236 (80, $\left.\mathrm{M}^{+}\right), 205$ (bp), 176 (91), 151 (34). IR (ATR): $\left(\mathrm{cm}^{-1}\right) v 2949,2217,1718,1606,1508,1455,1374,1280$.

CAS registry number: 42497-80-3.

3.8.5. 1-Phenyl-2-(o-tolyl)acetylene (13e)

${ }^{1} \mathrm{H}$ NMR $\left(\mathrm{CDCl}_{3}\right) \delta 7.44-7.39(\mathrm{~m}, 3 \mathrm{H}), 7.25-7.21(\mathrm{~m}, 3 \mathrm{H}), 7.12-7.10(\mathrm{~m}, 2 \mathrm{H}), 7.08-7.04$ $(\mathrm{m}, 1 \mathrm{H}), 2.41(\mathrm{~s}, 3 \mathrm{H}):{ }^{13} \mathrm{C} \mathrm{NMR}\left(\mathrm{CDCl}_{3}\right) \delta 166.5,131.7,131.4,129.5,129.4,128.7,128.4,127.9$, 122.6, 92.4, 88.6, 52.9. MS (EI): $m / z$ (rel\%) 192 (bp, M ), 165 (27), 115 (11). IR (ATR): $\left(\mathrm{cm}^{-1}\right) v$ $3055,2919,1600,1492$.

CAS registry number: 14309-60-5. 
3.8.6. 1-Phenly-2-(m-tolyl)acetylene (13f)

${ }^{1} \mathrm{H} \mathrm{NMR}\left(\mathrm{CDCl}_{3}\right) \delta 7.51(\mathrm{~d}, J=5.8 \mathrm{~Hz}, 2 \mathrm{H}), 7.35-7.28(\mathrm{~m}, 5 \mathrm{H}), 7.21(\mathrm{t}, J=7.5 \mathrm{~Hz}, 1 \mathrm{H}), 7.12(\mathrm{~d}$, $J=7.5 \mathrm{~Hz}, 1 \mathrm{H}), 2.33(\mathrm{~s}, 3 \mathrm{H}):{ }^{13} \mathrm{C} \mathrm{NMR}\left(\mathrm{CDCl}_{3}\right) \delta 137.9,132.1,131.5,129.3,128.6,128.2,128.2$, 128.1, 123.3, 122.9, 89.5, 89.0, 21.1. MS (EI): $m / z$ (rel\%) 192 (bp M $\left.{ }^{+}\right), 165$ (13), 115 (5). IR (ATR): $\left(\mathrm{cm}^{-1}\right)$ v 3003, 2919, 1492.

CAS registry number: $14635-91-7$.

3.8.7. 1-(1-Naphthyl)-2-phenylacetylene (13g)

${ }^{1} \mathrm{H}$ NMR $\left(\mathrm{CDCl}_{3}\right) \delta 8.44(\mathrm{~d}, J=8.3 \mathrm{~Hz}, 1 \mathrm{H}), 7.83(\mathrm{ddd}, J=13.2,13.2,4.9 \mathrm{~Hz}, 2 \mathrm{H}), 7.75(\mathrm{~d}$, $J=7.1 \mathrm{~Hz}, 1 \mathrm{H}), 7.64(\mathrm{~d}, J=7.3 \mathrm{~Hz}, 2 \mathrm{H}), 7.58(\mathrm{t}, J=7.3 \mathrm{~Hz}, 1 \mathrm{H}), 7.52(\mathrm{t}, J=7.3 \mathrm{~Hz}, 1 \mathrm{H}), 7.44(\mathrm{t}$, $J=7.6 \mathrm{~Hz}, 1 \mathrm{H}), 7.39-7.34(\mathrm{~m}, 3 \mathrm{H}):{ }^{13} \mathrm{C} \mathrm{NMR}\left(\mathrm{CDCl}_{3}\right) \delta 133.1,131.7,131.6,130.3,128.7,128.4$, 128.3, 128.2, 128.2, 126.7, 126.4, 126.1, 125.2, 125.2, 94.3, 87.5. MS (EI): $m / z(\mathrm{rel} \%) 228\left(\mathrm{bp}, \mathrm{M}^{+}\right)$, 202 (6), 113 (13). IR (ATR): $\left(\mathrm{cm}^{-1}\right) v$ 3055, 1488, 1396.

CAS registry number: 4044-57-9.

3.8.8. 1-(o-chlorophenyl)-2-phenylacetylene (13h)

${ }^{1} \mathrm{H}$ NMR $\left(\mathrm{CDCl}_{3}\right) \delta$ 7.57-7.55 (m, $\left.3 \mathrm{H}\right), 7.53-7.50(\mathrm{~m}, 1 \mathrm{H}), 7.34-7.32(\mathrm{~m}, 3 \mathrm{H}), 7.22-7.18(\mathrm{~m}, 2$ $\mathrm{H}):{ }^{13} \mathrm{C} \mathrm{NMR}\left(\mathrm{CDCl}_{3}\right) \delta 135.9,133.2,131.7,129.2,128.6,128.3,126.4,123.1,122.8,94.5,86.2 . \mathrm{MS}$ (EI): $m / z(\mathrm{rel} \%) 212$ (bp, M$\left.{ }^{+}\right), 176$ (38), 151 (11). IR (ATR): $\left(\mathrm{cm}^{-1}\right) v 3057,1491,1468$.

CAS registry number: 10271-57-5.

3.8.9. 1-(m-chlorophenyl)-2-phenylacetylene (13i)

${ }^{1} \mathrm{H}$ NMR $\left(\mathrm{CDCl}_{3}\right)$ 8 7.46-7.43 (m, $\left.3 \mathrm{H}\right), 7.34-7.32(\mathrm{~m}, 1 \mathrm{H}), 7.29-7.27$ (m, $\left.3 \mathrm{H}\right), 7.23-7.16(\mathrm{~m}, 2$ $\mathrm{H}):{ }^{13} \mathrm{C} \mathrm{NMR}\left(\mathrm{CDCl}_{3}\right) \delta 134.1,131.6,131.4,129.6,129.5,128.5,128.4,128.3,124.9,122.7,90.5$, 87.8. MS (EI): $m / z$ (rel\%) 212 (bp, $\left.\mathrm{M}^{+}\right), 176$ (46), 151 (13). IR (ATR): $\left(\mathrm{cm}^{-1}\right) v 3055,1490$.

CAS registry number: 51624-34-1.

\subsection{Tsuji-Trost Coupling Reaction}

The general procedure to obtain Tsuji-Trost reaction products $\mathbf{1 6 a - d}$ is as described here for 16a. 1,3-Diphenyl-2-propenyl acetate $(\mathbf{1 4 a} ; 100 \mathrm{mg}, 0.4 \mathrm{mmol})$ was added to a mixture of polymeric catalyst $(\mathbf{1} ; 80 \mathrm{mg}, 0.024 \mathrm{mmol})$, and sodium azide $(\mathbf{1 5} ; 39 \mathrm{mg}, 0.6 \mathrm{mmol})$ in $\mathrm{H}_{2} \mathrm{O}(3.0 \mathrm{~mL})$. The reaction mixture was shaken at $70{ }^{\circ} \mathrm{C}$ for $24 \mathrm{~h}$ and then filtered. The recovered resin beads were rinsed with $\mathrm{H}_{2} \mathrm{O}$ and extracted three times with EtOAc $(6 \mathrm{~mL})$. The EtOAc layer was separated and the aqueous layer was extracted with EtOAc $(3 \mathrm{~mL})$. The combined EtOAc extracts were washed with brine ( $2 \mathrm{~mL}$ ), dried over $\mathrm{MgSO}_{4}$, and concentrated in vacuo. The resulting residue was chromatographed on silica gel (hexane) to give $72.7 \mathrm{mg}$ (77\% yield) of 1,3-diphenyl-2-propenyl azide (16a). 


\subsection{Spectral and Analytical Data for the Allyl Azide}

3.10.1. 1,3-Diphenyl-2-propenyl azide (16a)

${ }^{1} \mathrm{H}$ NMR $\left(\mathrm{CDCl}_{3}\right) \delta 7.41-7.23(\mathrm{~m}, 10 \mathrm{H}), 6.71(\mathrm{~d}, J=15.6 \mathrm{~Hz}, 1 \mathrm{H}), 6.28(\mathrm{dd}, J=15.6,7.3 \mathrm{~Hz}, 1 \mathrm{H})$, $5.20(\mathrm{~d}, J=7.3 \mathrm{~Hz}, 1 \mathrm{H}):{ }^{13} \mathrm{C} \mathrm{NMR}\left(\mathrm{CDCl}_{3}\right) \delta 138.6,135.9,133.0,128.8,128.7,128.3,128.2,127.1$, 126.9, 126.8, 67.2. MS (EI): $\mathrm{m} / z$ (rel\%) 207 (58, M+), 193 (bp), 130 (40), 115 (85). IR (ATR): $\left(\mathrm{cm}^{-1}\right)$ v 3028, 2093, 1882, 1809.

CAS registry number: 120990-01-4.

\subsubsection{Cinnamyl azide (16b)}

${ }^{1} \mathrm{H} \mathrm{NMR}\left(\mathrm{CDCl}_{3}\right) \delta 7.39(\mathrm{~d}, J=7.3 \mathrm{~Hz}, 2 \mathrm{H}), 7.32(\mathrm{t}, J=7.3 \mathrm{~Hz}, 2 \mathrm{H}), 7.26(\mathrm{t}, J=7.3 \mathrm{~Hz}, 1 \mathrm{H})$, $6.63(\mathrm{~d}, J=15.8 \mathrm{~Hz} 1 \mathrm{H}), 6.23(\mathrm{td}, J=6.7,15.8 \mathrm{~Hz}, 1 \mathrm{H}), 3.92(\mathrm{~d}, J=6.7 \mathrm{~Hz}, 2 \mathrm{H}):{ }^{13} \mathrm{C} \mathrm{NMR}\left(\mathrm{CDCl}_{3}\right)$ $\delta$ 135.9, 134.4, 128.6, 128.1,126.5, 122.3, 52.9. IR (ATR): $\left(\mathrm{cm}^{-1}\right) v 3061,2927,2092$.

CAS registry number: 28022-21-1.

\subsubsection{3-(p-Tolyl)allyl azide (16c)}

${ }^{1} \mathrm{H}$ NMR $\left(\mathrm{CDCl}_{3}\right) \delta 7.29(\mathrm{~d}, J=8.0 \mathrm{~Hz}, 2 \mathrm{H}), 7.14(\mathrm{~d}, J=8.0 \mathrm{~Hz}, 2 \mathrm{H}), 6.61(\mathrm{~d}, J=15.8 \mathrm{~Hz}, 1 \mathrm{H})$, $6.18(\mathrm{td}, J=6.7,15.2 \mathrm{~Hz} 1 \mathrm{H}), 3.92(\mathrm{~d}, J=6.1 \mathrm{~Hz}, 2 \mathrm{H}), 2.33(\mathrm{~s}, 3 \mathrm{H}):{ }^{13} \mathrm{C} \mathrm{NMR}\left(\mathrm{CDCl}_{3}\right) \delta 138.1$, 134.5, 133.2, 129.3, 126.5, 121.2, 53.1, 21.2. MS (EI): $\mathrm{m} / \mathrm{z}$ (rel\%) 173 (10, M+), 144 (63), 131 (bp), 116 (23). HRMS (EI): calcd for $\mathrm{C}_{10} \mathrm{H}_{11} \mathrm{~N}_{3}$ 173.0953, found 173.0960. IR (ATR): $\left(\mathrm{cm}^{-1}\right) v 3026,2922$, $1652,1512$.

3.10.4. 3-(p-Trifluoromethylphenyl)allyl azide (16d)

${ }^{1} \mathrm{H}$ NMR $\left(\mathrm{CDCl}_{3}\right) \delta 7.58(\mathrm{~d}, J=7.9 \mathrm{~Hz}, 2 \mathrm{H}), 7.48(\mathrm{~d}, J=7.9 \mathrm{~Hz}, 2 \mathrm{H}), 6.67(\mathrm{~d}, J=15.8 \mathrm{~Hz}, 1 \mathrm{H})$, $6.32(\mathrm{td}, J=6.1,15.8 \mathrm{~Hz} 1 \mathrm{H}), 3.98(\mathrm{~d}, J=6.1 \mathrm{~Hz}, 2 \mathrm{H}):{ }^{13} \mathrm{C} \mathrm{NMR}\left(\mathrm{CDCl}_{3}\right) \delta 139.4,132.3,129.9$ (q, $J=33.0 \mathrm{~Hz}), 126.7,125.6(\mathrm{q}, J=4.1 \mathrm{~Hz}), 125.2,124.0(\mathrm{q}, J=270 \mathrm{~Hz}), 52.6 . \mathrm{MS}(\mathrm{EI}): \mathrm{m} / z(\mathrm{rel} \%) 227$ (23, M+), 206 (42), 185 (bp). HRMS (EI): calcd for $\mathrm{C}_{10} \mathrm{H}_{8} \mathrm{~F}_{3} \mathrm{~N}_{3}$ 227.0670, found 227.0674. IR (ATR): $\left(\mathrm{cm}^{-1}\right) v$ 2099, 1321. 227.06703.

\subsection{Recycling Experiments for Cross-Coupling Reaction}

The general procedure for recycling experiments to obtain cross-coupling coupling reaction products is as described here for 13a (Suzuki-Miyaura coupling product). To a mixture of the polymeric catalyst 1 (80 mg, $0.020 \mathrm{mmol}), \mathrm{K} 2 \mathrm{CO} 3(110 \mathrm{mg}, 0.8 \mathrm{mmol})$, and iodobenzene (7a; $81 \mathrm{mg}$, $0.4 \mathrm{mmol})$ in $\mathrm{H}_{2} \mathrm{O}(3.0 \mathrm{~mL})$ was added phenylboronic acid $(8 \mathbf{a} ; 97 \mathrm{mg}, 0.8 \mathrm{mmol})$. The reaction mixture was shaken at $100{ }^{\circ} \mathrm{C}$ for $6 \mathrm{~h}$ and filtered. The recovered resin beads were rinsed with $\mathrm{H}_{2} \mathrm{O}$ and extracted three times with EtOAc $(6 \mathrm{~mL})$. The EtOAc layer was separated and the aqueous layer was extracted with EtOAc $(3 \mathrm{~mL})$. The combined EtOAc extracts were washed with brine $(2 \mathrm{~mL})$, dried 
over $\mathrm{MgSO}_{4}$, and concentrated in vасио. The resulting residue was chromatographed on silica gel (hexane) to give $57 \mathrm{mg}$ (93\% yield) of biphenyl (9a). After first rus, the recovered resin beads were dried in vacuo and directly applied in the next reaction under similar conditions. The reaction of iodobenzene (7a; $81 \mathrm{mg}, 0.4 \mathrm{mmol})$ and phenylboronic acid (8a; $48 \mathrm{mg}, 0.4 \mathrm{mmol})$ with the recovered catalyst 1 took place in $\mathrm{K} 2 \mathrm{CO} 3(110 \mathrm{mg}, 0.8 \mathrm{mmol})$ aqueous solution $(3.0 \mathrm{~mL})$ to give the biphenyl in $57 \mathrm{mg}$ (93\% yield) of biphenyl (9a). For second and third runs, these reaction conditions and procedures were also the same as first reuse procedures.

\section{Conclusions}

We developed a novel polymer-supported terpyridine palladium complex through ionic bonds to a sulfonate group, which efficiently catalyzed the Suzuki-Miyaura, Mizoroki-Heck, Sonogashira, and Tsuji-Trost, cross-coupling reactions in water under aerobic and mild reaction conditions to give the corresponding cross-coupling product with a high to excellent yield. This catalyst was recovered and reused several times without any loss of catalytic activity. Efforts to further extend the scope of the cross-coupling reactions and the application of this catalyst for other organic transformations are in progress in our lab.

\section{Acknowledgements}

We are grateful for the financial support from the Cooperative Research Program of Institute for Materials Chemistry and Engineering, Kyushu University. This work was the Joint Study Program of the Institute for Molecular Science. We also thank the Cooperative Research Center for their financial support for this work.

\section{References and Notes}

1. Trost, B.M. The atom economy-A search for synthetic efficiency. Science 1991, 254, 1471-1477.

2. Tsuji, J. Palladium Reagents and Catalysis: Innovations in Organic Synthesis; Wiley: New York, NY, USA, 1995.

3. Yin, L.; Liebscher, J. Carbon-carbon coupling reactions catalyzed heterogeneous palladium catalysts. Chem. Rev. 2007, 107, 133-173.

4. Tsuji, J. Palladium Reagents and Catalysts; Wiley: New York, NY, USA, 2004.

5. Heck, R.F. Palladium Reagents in Organic Synthesis; Academic: London, UK, 1985.

6. Tsuji, J.; Takahashi, H.; Morikawa, M. Organic synthesis by means of noble metal compounds XVII. Reaction of $\pi$-allylpalladium chloride with nucleophiles. Tetrahedron Lett. 1965, 6, 4387-4388.

7. Trost, B.M.; Fullerton, T.J. New synthetic reactions allylic alkylation. J. Am. Chem. Soc. 1973, 95, 292-294.

8. Mizoroki, T.; Mori, K.; Ozaki, A. Arylation of olefin with aryl iodine catalyzed by palladium. Bull. Chem. Soc. Jpn. 1971, 44, 581-581.

9. Heck, R.F.; Nolly, J.P. Palladium-catalyzed vinylic hydrogen substitution reactions with aryl, benzyl, and styryl halides. J. Org. Chem. 1972, 37, 2320-2322. 
10. Miyaura, N.; Yanagi. T.; Suzuki, A. The palladium-catalyzed cross-coupling reaction of phenylboronic acid with haloarenes in the presence of bases. Synth. Commun. 1981, 11, 513-519.

11. Sonogashira, K.; Tohda, Y.; Hagihara, N. Convenient synthesis of acetylenes. Catalytic substitutions of acetylenic hydrogen with bromo alkenes, iodo arenes and bromopyridines. Tetrahedron Lett. 1975, 16, 4467-4470.

12. Beletskaya, I.P.; Cheprakov, A.V. The Heck reaction as a sharpening stone of palladium catalysis. Chem. Rev. 2000, 100, 3009-3066.

13. Chinchilla, R.; Nájera, C. The Sonogashira reaction: A booming methodology in synthetic organic chemistry. Chem. Rev. 2007, 107, 874-922.

14. Miyaura, N.; Suzuki, A. Palladium-catalyzed cross-coupling reactions of organoboron compounds. Chem. Rev. 1995, 95, 2457-2483.

15. Trost, B.M.; Crawley, M.L. Asymmetric transition-metal-catalyzed allylic alkylations: Application in total synthesis. Chem. Rev. 2003, 103, 2921-2944.

16. Alonso, F.; Beletskaya, I.P.; Yus, M. Non-conventional methodologies for transition-metal catalyzed carbon-carbon coupling: A critical overview. Tetrahedron 2005, 61, 11771-11835.

17. Yin, L.; Liebscher, J. Carbon-carbon coupling reactions catalyzed by heterogeneous palladium catalysts. Chem. Rev. 2007, 107, 133-173.

18. For a review, see Sheldon, R.A.; I. Arends, U. Hanefeld, Green Chemistry and Catalysis; Wiley-VCH: Weinheim, Germany, 2007.

19. For a review, see Ding, K.; Uozumi, Y. Handbook of Asymmetric Heterogeneous Catalysis; Wiley-VCH: Weinheim, Germany, 2007.

20. Kann, K. Recent applications of polymer supported organometallic catalysts in organic synthesis. Molecules 2010, 15, 6306-6331.

21. Suzuka, T.; Ooshiro, K.; Kina, K. Reusable polymer-supported terpyridine copper complex for [3 + 2] Huisgen cycloaddition in water. Heterocycles 2010, 81, 601-610.

22. Suzuka, T.; Nagamine, T.; Ogihara, K.; Higa, M. Suzuki-Miyaura cross-coupling reaction in water with polymer-supported terpyridine palladium complex under aerobic conditions. Catal. Lett. 2010, 139, 85-89.

23. For a review of terpyridine-metal complexes, see Eryazici, I.; Moorefield, N.; Newkome, R. Square planar $\mathrm{Pd}(\mathrm{II}), \mathrm{Pt}(\mathrm{II})$, and $\mathrm{Au}(\mathrm{III})$ terpyridine complexes: Their synthesis, physical properties, supra-molecular constructs, and biomedical activities. Chem. Rev. 2008, 108, 1834-1895 and references therein.

24. For an example of immobilization of organometallic catalyst onto the solid-support through ionic bonds, see Uthaiwan, S.; Jin, B.Y.; Se, L.B.; Yoon, C.D. Ionic polymer supported copper(I): A reusable catalyst for Huisgen's 1,3-diopar cycloaddition. Synlett 2008, 40, 2326-2330.

25. Horváth, I. Hydroformylation of olefins with the water soluble $\mathrm{HRh}(\mathrm{CO})\left[\mathrm{P}\left(\mathrm{m}-\mathrm{C}_{6} \mathrm{H}_{4} \mathrm{SO}_{3} \mathrm{Na}\right)_{3}\right]_{3}$ in supported aqueous-phase. Is it really aqueous? Catal. Lett. 1990, 6, 43-48. 
26. For control experiments for entry 1 in Table 1 ; 1) the Suzuki-Miyaura cross-coupling reaction of phenylboronic acids with iodobenzene was preformed with $5 \mathrm{~mol} \%$ of $\mathrm{Pd}\left(\mathrm{NCCH}_{3}\right)_{2} \mathrm{Cl}_{2}$ and PS-PEG-NH $\mathrm{NH}_{2}$ resin at $100{ }^{\circ} \mathrm{C}$ for $6 \mathrm{~h}$ to give the biphenyl in $33 \%$ yield. ICP-AES analysis of the filtrate showed that the Pd content was $2.2 \mathrm{ppm}$.

(C) 2011 by the authors; licensee MDPI, Basel, Switzerland. This article is an open access article distributed under the terms and conditions of the Creative Commons Attribution license (http://creativecommons.org/licenses/by/3.0/). 